\title{
From Special Least-Squares to Twin Laws: My Toolbox of Programs from 1964 to $2017 .{ }^{1}$
}

\author{
Bruce M. Foxman
}

Department of Chemistry, MS015, Brandeis University, Waltham, MA 02453-2700

As a graduate student in the 1960s, the need to have data processing, weighting analysis and rigid-body least-squares programs at MIT provided the incentive to learn FORTRAN and IBM 7094-assembler language, and led to a lifetime interest in solving problems using simple-to-complex programs. Our earliest post-Ph. D. success, a collaboration with Mike Bennett and Wendy Hutcheon, was a leastsquares program to model the scattering from hindered rotors in crystals. ${ }^{2}$ Over the years, independent programs and/or 'transfer-from-mainframe-to-PC' exercises were common: for example, Weiss, a program to draw Weissenberg-method festoons, and Tracer-IIPC, a port of Steve Lawton's very pretty cell-reduction program to the PC. At recent ACA meetings and elsewhere, we have presented our analysis of the alignment of mother and daughter phases using the local program TOPO, currently available as a download from http://www.xray.chem.brandeis.edu. ${ }^{3}$ A recent dialogue with llia Guzei is leading to useful updates to TOPO, soon to be released. The analysis of phase transitions and twinning effects using TOPO prompted us to learn and develop our knowledge of twin laws, and the relationship between obliquity and the actual twins commonly observed in molecular crystals. For this we needed a copy of Yvon Le Page's program OBLIQUE, for which the code and executables are unavailable. We were fortunate enough to have some stimulating discussions with Professor Howard Flack, who provided a copy of CREDUC81 by Le Page. Studying the CREDUC81 code led us to write our own code, which provides an improved analysis of the twin laws suggested by OBLIQUE. The current status of that project will be discussed, along with some future post-retirement projects, including an upcoming effort for the preservation and use of the CAD4-PC code as a teaching tool.

1. This presentation is dedicated to the memory of Professor Howard D. Flack.

2. Bennett, M. J.; Hutcheon, W. L.; Foxman, B. M. Acta Crystallogr., Sect. A 1975, A31, 488-494.

3. Posner, S. R.; Lorson, L. C.; Gell, A. R.; Foxman, B. M. Cryst. Growth Des. 2015, 15, 3407-3416. 